\title{
An Integrated Framework for Designing Content based Image Retrieval System using Support Vector Machine with Enhanced Biased Maximum Margin
}

\author{
Neera Lal \\ Research Scholar, IT Dept \\ TIT, \\ Bhopal, India
}

\author{
Neetesh Gupta \\ Professor, IT Dept \\ TIT, \\ Bhopal, India
}

\author{
Amit Sinhal \\ Professor, IT Dept \\ TIT, \\ Bhopal, India
}

\begin{abstract}
With the growth of new era and development a wide variety of applications are being developed in the field of CBIR. There are different applications of relevance feedback approaches to improve the performance of image retrieval. The main concept of using relevance feedback is to overcome semantic gap between low level features and high level concepts. Among the most popular approaches to RF support vector machine is the key to interactive image retrieval. The reason to employ SVM with RF is to make image retrieval more efficient. Despite of the fact that SVM improves the performance efficient methods and techniques are still needed to be developed that can efficiently retrieve more relevant images. The main problem with the RF approach with SVM is it considers positive and negative samples equally. The two samples are different in their properties and cannot be treated equally. In this paper we will present enhanced biased maximum margin support vector machine. In this method negative samples of RF approach are efficiently sampled by shared nearest neighbor and it is optimized by ant colony optimizer with biased maximum margin. This concept mainly maps positive samples closer and negative samples are distinguished from them by maximum margin. It thus improves the performance by bringing most relevant images nearer less relevant images farer.
\end{abstract}

\section{KEYWORDS}

Content based image retrieval, relevance feedback, support vector machine, shared nearest neighbor, ant colony optimization.

\section{INTRODUCTION}

With the immense growth and development of World Wide Web data including multimedia concept like videos images and many more has gathered substantial attention in day to day life. The most promising and popular fundamental area of one' $s$ interest is CBIR with a lot of many potential practical applications[1]. Content Based image Retrieval is the main concept to retrieve the image that matches some criteria used to search images. For the image retrieval a query image is being provided and the system will return the image similar to query image. But due to the development of computer network and minimum cost of storage devices visual information is mainly used in many fields. The retrieval of visual features efficiently has given rise to new evolution of different techniques of retrieving images. Image retrieval systems allow organized querying searching and browsing by image content[3]. But the researches in the early years only explained best representation of visual features. It is just only based on similarity of images and adding distances of low level features with defined weights. It does not take into consideration high level features and human perception. This method only degrades the performance of CBIR[4]. So there was need of mechanism that takes into account to bridge the semantic gap between low level features and high level concepts also considers applicability to human perception.

- Relevance feedback - is a technique that considers human interaction to refine high level concepts defined by low level features. It is basically used in text based retrieval[5]. In the application of using relevance feedback first query image is selected then user selects or marks the relevant images from retrieved result and modifies the query. It also provides the preference weights for relevant images. Based on the user feedback high level concepts defined by the query weights automatically refined[6].

- Support vector machine- is an implementation of structural risk minimization principle. It builds a classifier with minimized Vapnik Chervonenkis dimension. Given a set of linearly separable samples $\left(x_{i}\right.$ ,$\left.y_{i}\right), \quad x_{i} \in R^{d}, \quad y_{i} \in\{-1,1\}$ is the class label which $x_{i}$ belongs to[7]. The general form of linear classification is $\mathrm{g}(\mathrm{x})=\mathrm{w} \cdot \mathrm{x}+\mathrm{b}$, which defines the hyper plane $\mathrm{w} \cdot \mathrm{x}$ $+\mathrm{b}=0$.

- Shared nearest neighbor - is a density based algorithm defined by Jarvis [8] and improved for other purposes too. It is a density based algorithm, mainly SNN method focuses on how to cluster objects based on high density parts. The basic idea is that 
extent to which more two objects share a common neighbor; these objects must appear more in the cluster. In the SNN graph, the strength of a link is the number of shared neighbors between the objects [9].

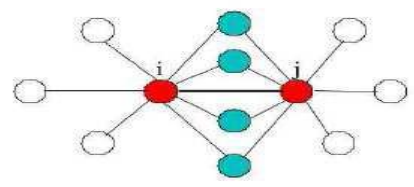

Figure 1. Object $\mathbf{i}$ and $\mathbf{j}$ are linked together with four shared neighbors

- Ant Colony optimization - concept was conceived by Marco Dingo in 1992. It is based on foraging behavior of ants. The ACO is most powerful optimization technique in the field of swarm intelligence. The concept of ACO resides in colony brain communication done by the pheromone[10]. Ant uses pheromone as a chemical messenger.

- Biased maximum margin - It is mainly used to map positive samples i.e. relevant images closer and negative samples i.e. irrelevant images are separated from positive ones by maximum margin in the feature space. In the feature space negative samples that are different in some aspects with the query image are distinguished from positive samples by a maximum margin which have similar concept to query image. So it is easy to map relevant and irrelevant images onto feature space[24]. (a) (b)

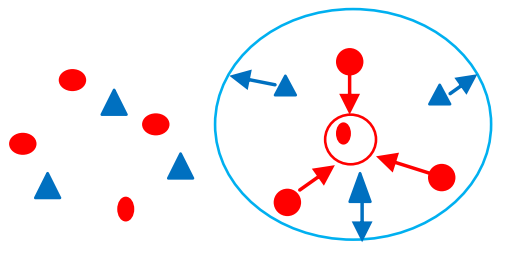

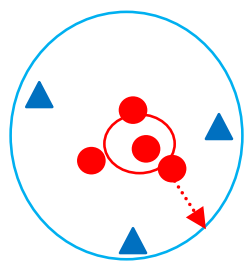

(c)
Figure 2. (a) Red dots are positive samples; blue dots are negative samples (b) BMM method (c) Positive \& negative samples in margin space

\section{RELATED WORK}

Mainly RGB color is used color retrieval. As RGB color space do not provide all the color information HSV color space is used.

- Color feature - Every image comprises of three primary colors namely red, blue and green. In this paper HSV color space is used. HSV stands for hue, saturation, and value.

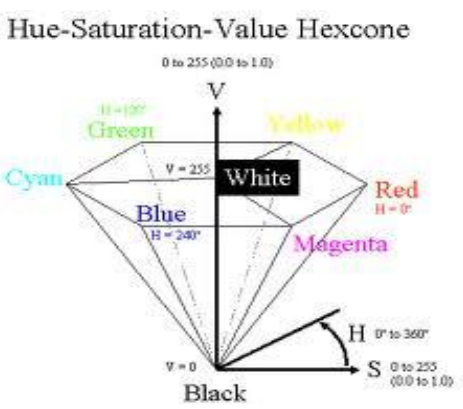

Figure: 3. (a) HSV coordinate system [11]

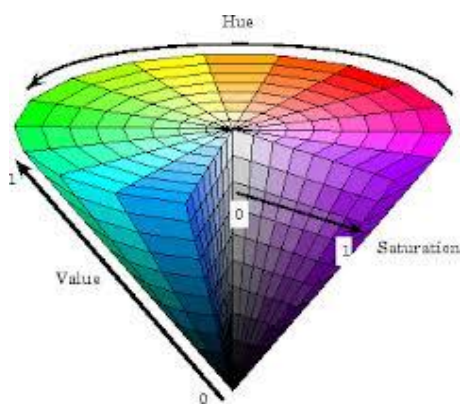

Figure: 3. (b) HSV model [11]

Hue describes the actual wavelength of the color. Saturation is measure of purity of color. It shows how much of white light is added [11].

- Texture feature- It is another important feature of an image. But there is no precise definition for texture. Texture can be defined as the visual patterns that have the property of homogeneity [24]. In this paper texture is calculated by gray level co- occurrence matrix. The energy function of an image is used. The energy function is defined by-

Energy $(\mathrm{E})=\sum_{i j} P(i, j)^{2} \quad-(1)$

Where $P(i, j)$ is the gray level co- occurrence matrix which is defined by specifying a displacement vector $\mathrm{d}$ and counting all pairs of pixels separated by $\mathrm{d}$ having gray level 0 and 1 .

- Shape feature- In this feature shape feature is extracted using sobel edge detector. The Sobel operator performs a 2-D spatial gradient measurement on an image. It is used to find the approximate absolute gradient magnitude at each point in an input grayscale image. The Sobel edge detector uses a pair of $3 \times 3$ convolution masks, one estimating the gradient in the $\mathrm{x}$-direction and the other estimating the gradient in the y-direction. The sobel operator uses masks to approximate digitally the first derivatives [26]. 


\section{PROPOSED WORK}

After reviewing all the approaches of image retrieving process there are some pros and cons. So there is a need of effective method to solve the problem. In this paper we have used biased maximum margin. Shared nearest neighbor is to map positive samples closer in maximum margin feature space. Ant colony optimizer is used optimize samples in selected feature space.

\section{Algorithm-}

1. In that phase feature extraction process is done.

2. Input image for the process.

3. Apply wavelet transform function and convert it into different layers.

4. Color extraction process -

i) Convert image from RGB to HSV.

ii) Calculate vector point

iii) Apply biased maximum margin with Gaussian kernel and perform training \& mapping

iv) Finally get the feature vector
5. Texture extraction process -

i) Convert image from $\mathrm{RGB}$ to grayscale.

ii) Apply energy function

iii) Calculate vector point

iv) Apply biased maximum margin with Gaussian kernel and perform training \& mapping.

v) Finally get the feature vector.

6. Shape extraction process -

i) Convert the image and perform removal of background

ii) Apply the sobel edge detector.

iii) Calculate the vector point

iv) Apply the biased maximum margin with Gaussian kernel and perform training \& mapping.

v) Finally get the feature vector.

7. All the feature vector go to feature space

8. Initialization of Ant for the selection of feature subset.

9. Check the feature space. If space is empty terminate the process of Ant. If not proceed.

10. Find the best score match

11. Images are retrieved (result)

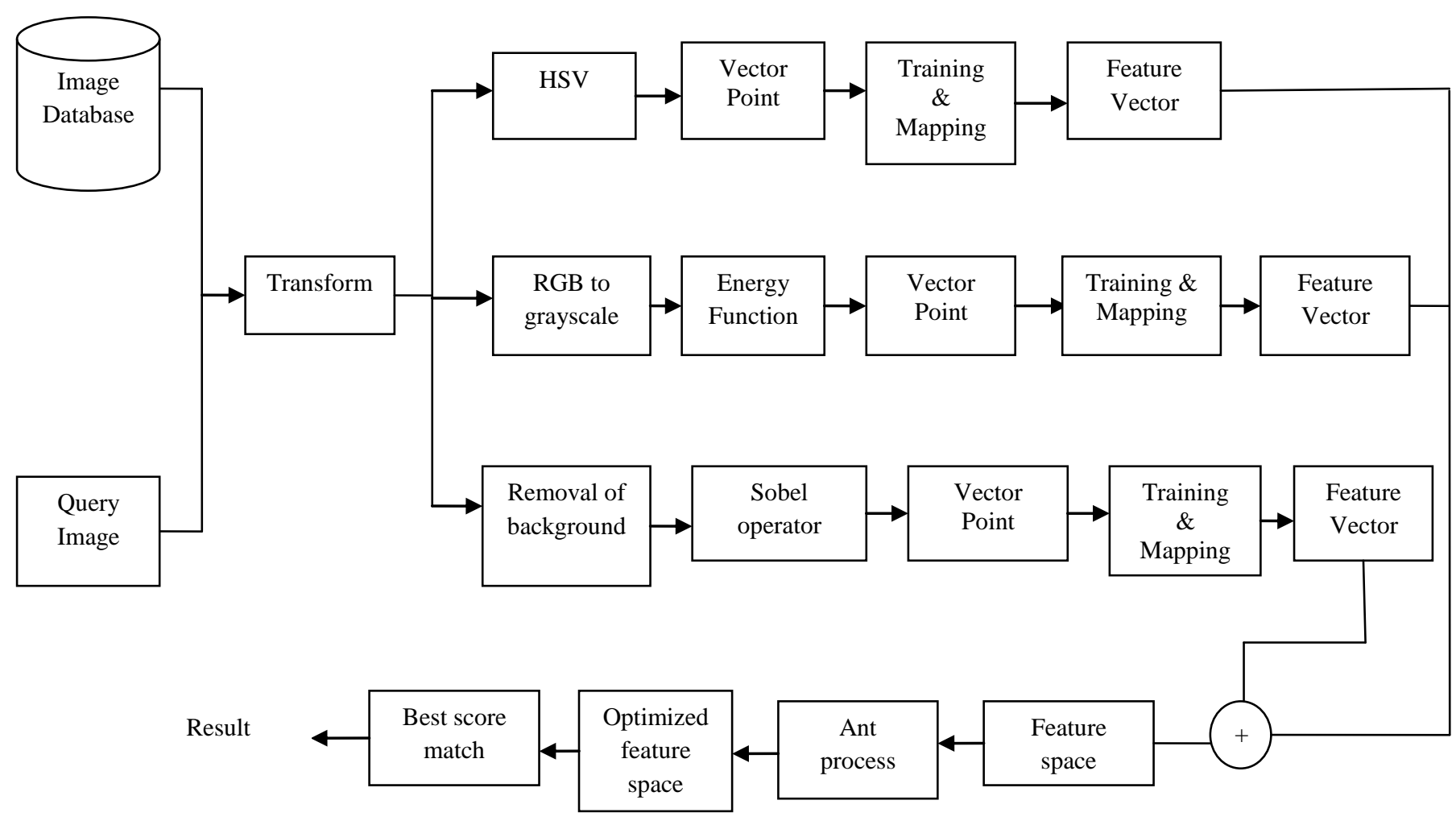

Figure: 4 Block Diagram of Proposed System 


\section{RESULT}

Initially feature extraction of all database images takes place. All feature vector created are stored separately. Firstly images to be taken are selected and then these images are required to update the record. Figure 5 shows the process.

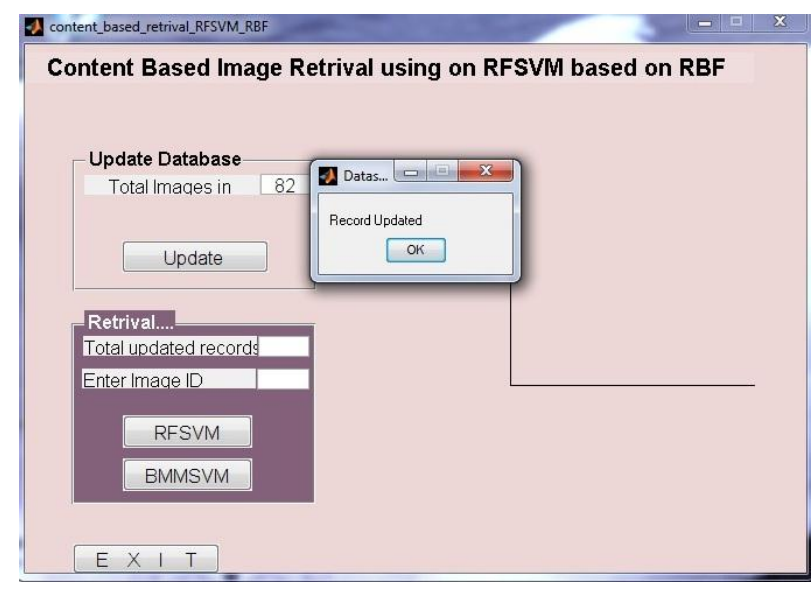

Figure 5: Database is being updated for the process with no. of images to be taken

After this query image is loaded. There are total 82 images belonging to ten different classes. The user only enters the image id and the result is displayed.

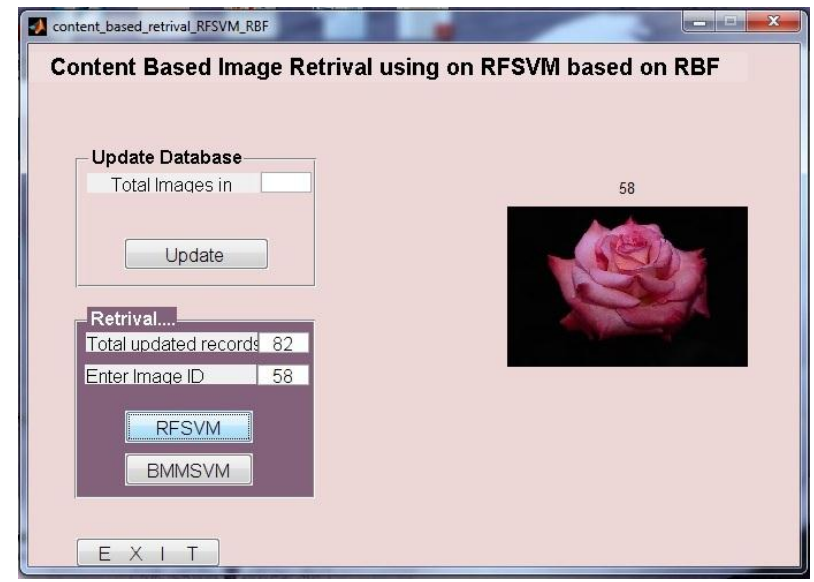

Figure 6: Query image is successfully loaded for the process

After the query image is successfully loaded retrieval is done using relevance feedback through support vector machine and the most similar images to the query image is displayed.

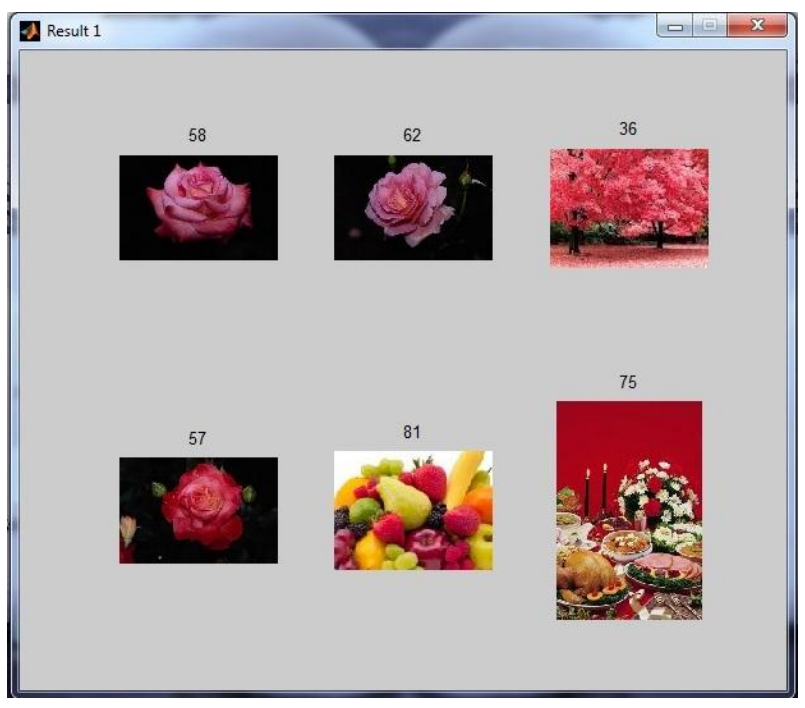

Figure 7(a): Result of RFSVM with most relevant images

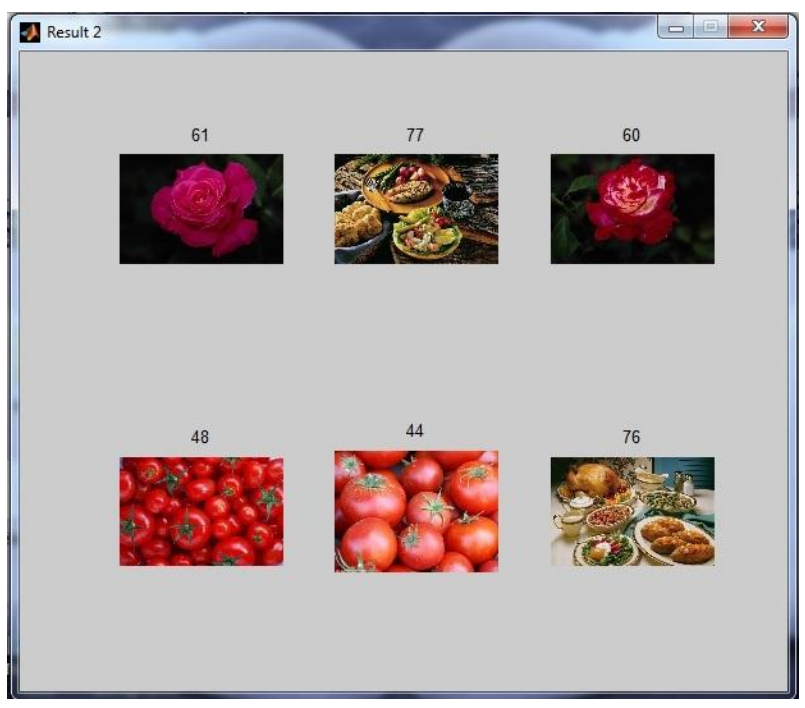

Figure 7(b): Result of RFSVM with less relevant images

The above figure shows the result of RFSVM. The top left image is the query image. Here it can be noted that the RF mechanism gives the relevant images. But also it treats the negative samples i.e. irrelevant images equally. In the top 12 images being displayed only 5 images are relevant and other not relevant. Also relevant images of roses are displayed between the negative samples. 


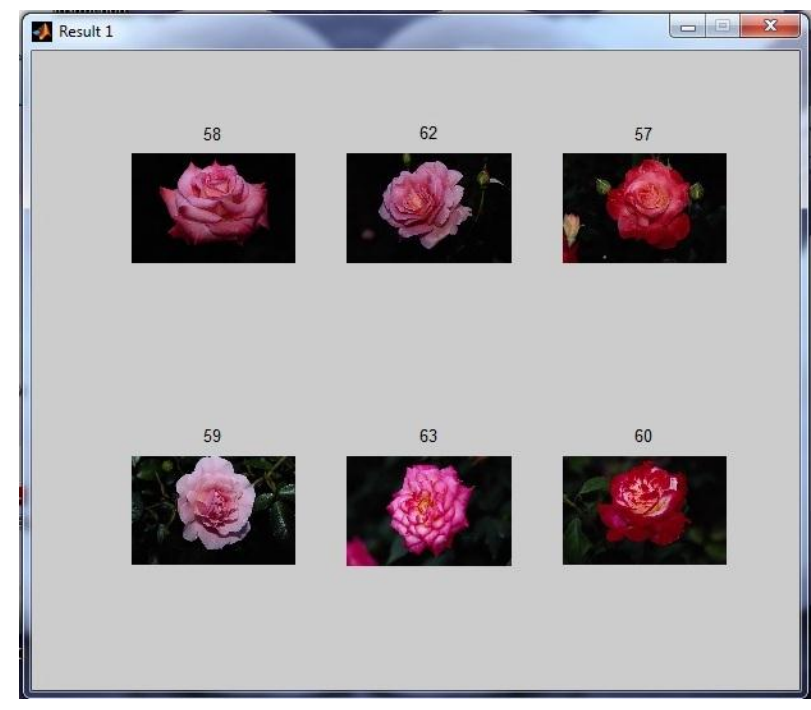

Figure 8(a): Result of EBMMSVM with most relevant images

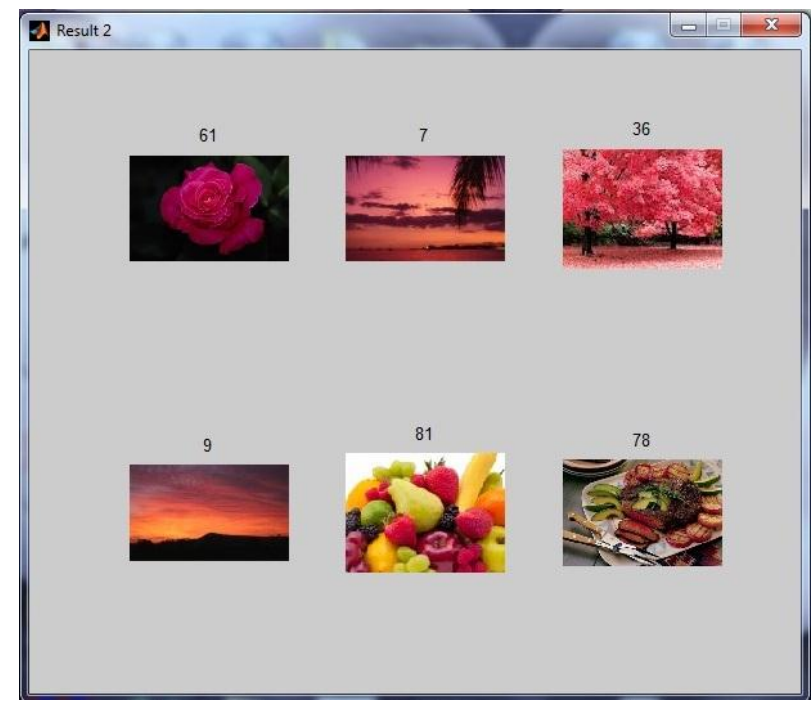

Figure 8(b): Result of EBMMSVM with less relevant images

The above figure shows the result of using biased maximum margin with support vector machine. Here the positive samples are differentiated by biased maximum margin. In this method 7 relevant images are retrieved. Now for performance of the algorithm can be evaluated by finding relevant images to the query image. The effectiveness and performance of the method can be determined by precision and recall rate.

Precision can be defined by the ratio of number of relevant images retrieved to total number of images retrieved. Recall can be defined by number of relevant image retrieved to total number images in the database.

Precision $=\frac{\text { No.of relevant } \text { images retrieved }}{\text { Total no. of } \text { images retrieved }}$
Recall $=\frac{\text { No.of relevant images retrieved }}{\text { Total no.of } \text { images in database }}$

Now the precision values are calculated for query image as well as for the whole category of the roses and then result is shown in table (a).

\begin{tabular}{|c|c|c|}
\hline Image id & RFSVM & BMMSVM \\
\hline 57 & 0.58 & 0.58 \\
\hline 58 & 0.416 & 0.58 \\
\hline 59 & 0.85 & 1 \\
\hline 60 & 0.85 & 1 \\
\hline 61 & 1 & 1 \\
\hline 62 & 1 & 1 \\
\hline 63 & 0.85 & 1 \\
\hline
\end{tabular}

Table (a): Precision values of both methods

The graph of the Table (a) is shown below.

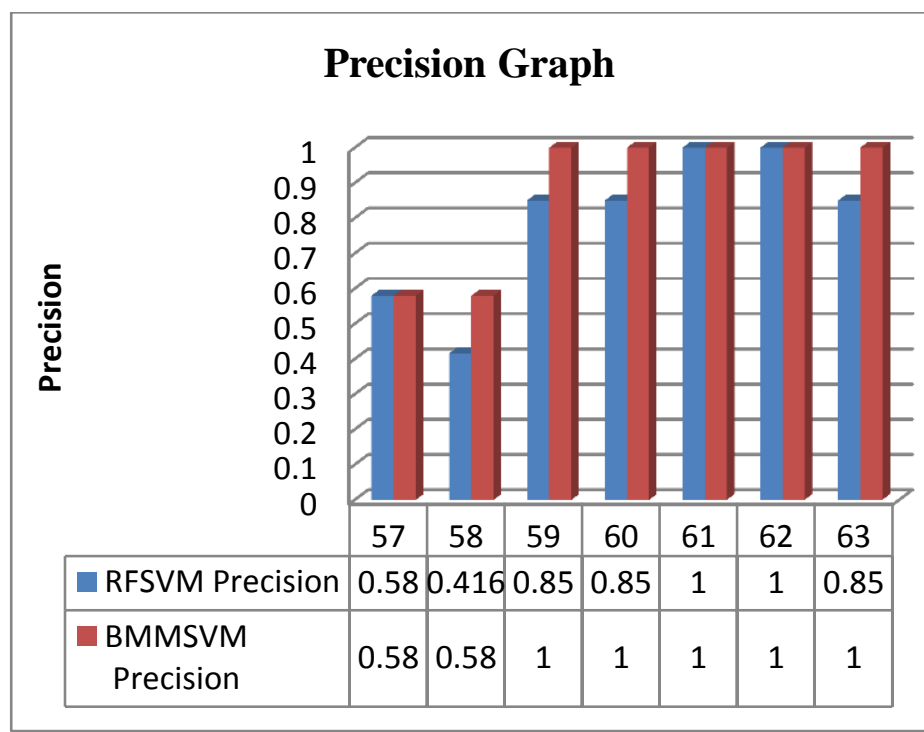

Figure 9 : Graph corresponding to table (a)

The recall values are calculated for the query image as well as for the whole category of roses and the result is shown in table(b).

\begin{tabular}{|c|c|c|}
\hline Image id & RFSVM & BMMSVM \\
\hline 57 & 1 & 1 \\
\hline 58 & 0.714 & 1 \\
\hline 59 & 0.85 & 1 \\
\hline 60 & 0.85 & 1 \\
\hline 61 & 1 & 1 \\
\hline 62 & 1 & 1 \\
\hline 63 & 0.85 & 1 \\
\hline
\end{tabular}

Table (b) : Recall values of both the methods 
The graph of table(b) is shown below.

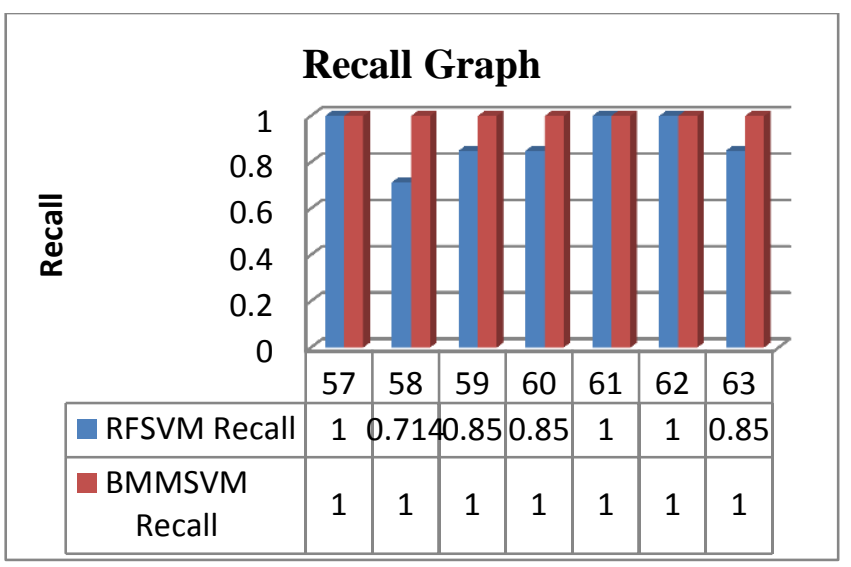

Figure 10: Graph corresponding to table (b)

The database contains ten images category of sunset, lion, elephants, autumn, tomato, dinosaur, rose, horse, mountains, and food. The proposed method is executed on all the images and then average values are calculated for each image category. The table of all categories is shown below. The figure: 11 shows performance of system using RFSVM.

\begin{tabular}{|c|c|c|c|}
\hline Category & $\begin{array}{c}\text { No. of } \\
\text { images }\end{array}$ & $\begin{array}{c}\text { Precision } \\
\%\end{array}$ & Recall \% \\
\hline Sunset & 11 & 76.48 & 83.61 \\
\hline Lion & 9 & 30.62 & 50.11 \\
\hline Elephants & 10 & 30.62 & 73 \\
\hline Autumn & 10 & 20.77 & 25 \\
\hline Tomato & 8 & 39.43 & 56.25 \\
\hline Dinosaur & 8 & 49.91 & 76.56 \\
\hline Rose & 7 & 52.22 & 74.12 \\
\hline Horse & 6 & 31.8 & 63.7 \\
\hline Mountains & 5 & 34.6 & 32 \\
\hline Food & 8 & 23 & 35 \\
\hline
\end{tabular}

Table (c): Precision and Recall based on RFSVM method

The graph of table (c) is shown below.

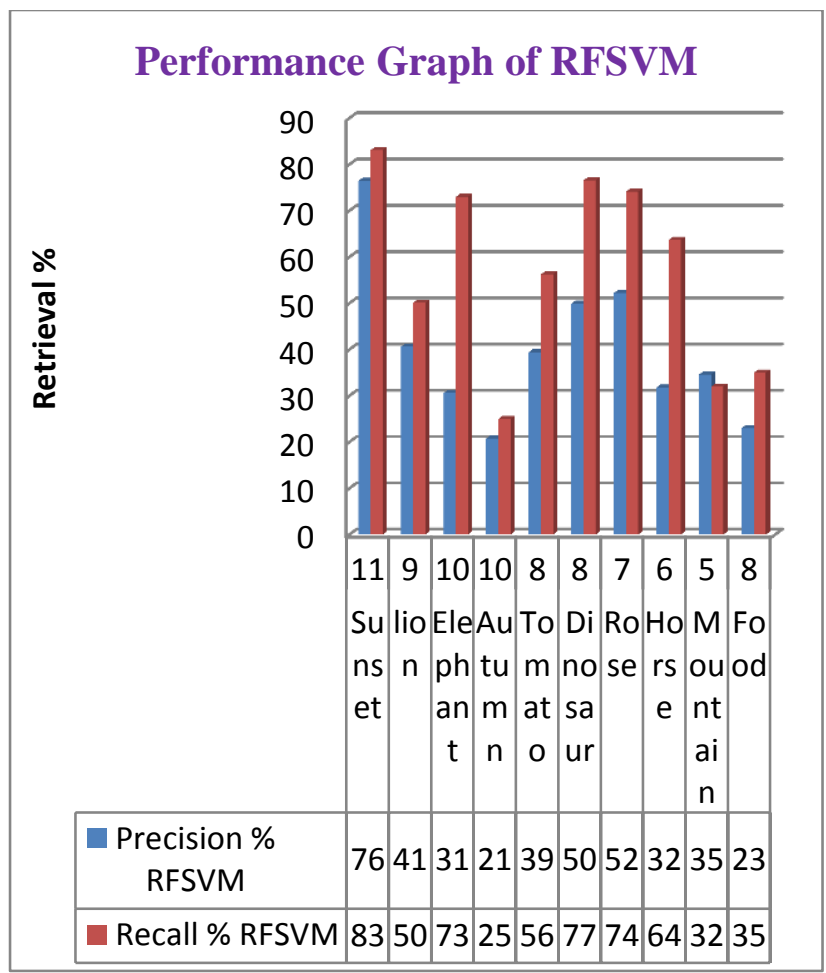

Figure 11: Graph corresponding to table (c)

The table (d) shows performance using the proposed method.

\begin{tabular}{|c|c|c|c|}
\hline Category & $\begin{array}{c}\text { No. of } \\
\text { images }\end{array}$ & $\begin{array}{c}\text { Precision } \\
\%\end{array}$ & Recall \% \\
\hline Sunset & 11 & 54.92 & 59.95 \\
\hline Lion & 9 & 44.35 & 58.66 \\
\hline Elephants & 10 & 54.75 & 66 \\
\hline Autumn & 10 & 40.78 & 49 \\
\hline Tomato & 8 & 53.88 & 81.25 \\
\hline Dinosaur & 8 & 60.15 & 77.03 \\
\hline Rose & 7 & 58 & 100 \\
\hline Horse & 6 & 47.1 & 94.3 \\
\hline Mountains & 5 & 36.2 & 88 \\
\hline Food & 8 & 33 & 46.8 \\
\hline
\end{tabular}

Table (d): Precision and recall based on Proposed method

The graph of table (d) is shown below. The figure 12 shows the performance of proposed method. 


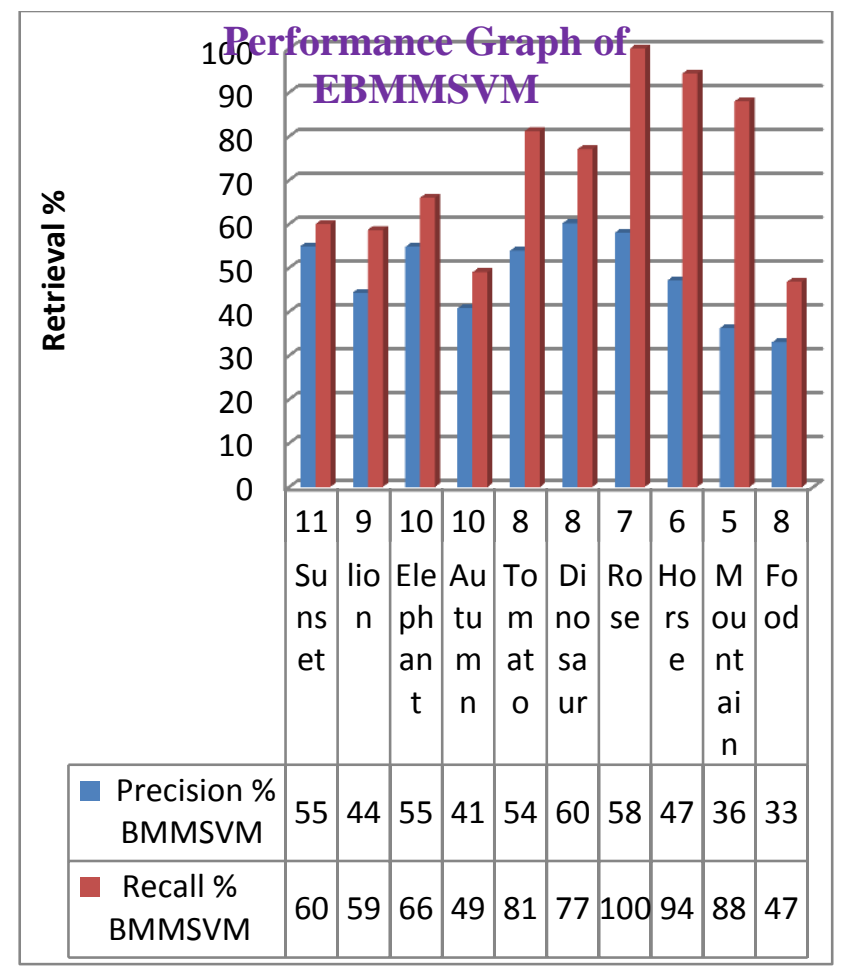

Figure 12: Graph corresponding to table (d)

\section{CONCLUSION AND FUTURE WORK}

In this paper, a new approach is presented with the help of enhanced biased maximum margin with support vector machine. As the previous approaches used with the relevance feedback suffers a major drawback. It treats positive and negative samples equally. As both the samples differ in their properties and semantics in the feature space. The biased maximum margin maps positive samples closer and negative samples are distinguished by maximum margin criteria. The sampling technique used i.e. SNN is used to create the subset of negative samples of RF technique. It is basically used map the samples in new feature space which have semantics as that of the query. The samples are now optimized by the ant colony optimizer to find the best score match with the query image. The HSV color model is used for the color feature. Energy function is used to find the texture feature. And lastly sobel operator is used to find the shape feature. Experimental results of the proposed system show the effectiveness of the method. And results in the improvement of the retrieval performance to major extent. In future work emphasis will be laid on investigating different technique of sampling other than SNN. It is time consuming process. So an effective technique is required to overcome the drawback. Secondly we will keep the record of all negative samples so that it can help in building a good classifier.

\section{REFERENCES}

[1] Ritendra Datta, Dhiraj Joshi, Jia Li, and James Wang Image Retrieval: Ideas, Influences, and Trends of New Age. In ACM Computing Surveys, Vol. 40. No.2, Article 5, 2008.

[2] Arnold W.M. Smeulders, Marcel Worrimg, Simone Santini, Amaranth Gupta, Ramesh Jain Content Based Image Retrieval at the End of Early Years. IEEE Transactions On Pattern Analysis and Machine Intelligence. Vol. 22, No.12, pp. 1349-1380, 2000.

[3] Zhi -Chun Huang, Patrick P.K. Chan, Wing W. Y. NG, Daniel S. Yeung CBIR using color moment and Gabor texture feature.

[4] Pengyu Hong, Qi Tian, Thomas S. Huang Incorporate SVM to CBIR with Relevance Feedback. In Proceedings of IEEE ICIP, Vol. 3, pp 750-753, 2000

[5] Peng Zhang, Jing Peng SVM vs. RLS classification. 2004.

[6] Y. Rui, T.S. Huang, et al ., Relevance Feedback: A Power Tool for Interactive Image Retrieval. IEEE Trans. on Circuits and Video Tech., 1998.

[7] Lei Zang, Fuzong Lin, Bo Zhang Support Vector Machine for Image Retrieval. pp. 721-724, 2001

[8] R.A. Jarvis, Edward A. Patrick Clustering Using a similarity measure based on SNN.

[9] Pierrie Alian Moellic, Jean Emnauel Haugeard, Guillaume Pittel Image Clustering based on SNN approach for tagged image collection

[10] Tomas Piatrik, Ebroul Izquierdo An application of Ant Colony optimization to image clustering.

[11] Youngeun An, Muhammad Riaz, Jongan Park CBIR based on adaptive segmentation of HSV color space. In $12^{\text {th }}$ International conference on Computer Modeling and Simulation, pp no. 248-251, 2010.

[12] Hui Yu, Mingling Li, Hong- Jiang, Jufu Feng Color Texture Moments for CBIR. In IEEE ICIP, pp no. 929-932, 2002.

[13] P.B. Thawari, N.J. Janwe CBIR based on Color and Texture. In IJITKM, Vol 4 No. 1, pp no. 129-132, 2011.

[14] Pushpa B. Patil, Manesh B. Kokare Interactive Content Based Texture Image Retrieval. In ICCCT pp no. 71-76, 2011.

[15] Chu-Hong Hoi and Michael Ri Lyu A Novel Log Based Relevance Feedback Technique in CBIR, ACM, pp 24-31, 2004.

[16] Dacheng Tao, Xiaoou Tang, Xuelong Li, Xindong Wu Asymmetric Bagging and Random Subspace for SVM based $\mathrm{RF}$ in Image retrieval. In IEEE Trans. on Pattern Analysis and Machine Intelligence, Vol. 28, No. 7, pp no. 1088-1099, 2006.

[17] Chu- Hong Hoi, Chi- Hang Chang, Kaizhu Huang, Michael R. Lyu and Irwin King Biased Support Vector Machine for Relevance Feedback in Image Retrieval. 
[18] Y. Lu, C. Hu, X. Zhu, H.J. Zhang, and Q. Yang A Unified Framework for Semantics and Feature Based Relevance Feedback in Image Retrieval Systems, ACM multimedia, 2000

[19] Yong Rui, Thomas S. Huang, Sharad Mehrotra RF Techniques in CBIR.

[20] Chu- Hong Hoi, Michael R. Lyu Group Based RF with SVM Ensembles.

[21] ] Dacheng Tao and Xiaoou Tang Random Sampling Based SVM for Relevance Feedback Image Retrieval. In Proceedings of IEEE Computer Society Conference on CVPR. 2004.

[22] Lei Wang, Yan Gao, Kap Luk Chan, Ping Xue, Wei-Yun Retrieval with knowledge driven kernel design: An Approach to Improving SVM based CBIR with RF. In
Proceedings of Tenth IEEE International Conference on Computer Vision 2005.

[23] Haifeng Li, Jiang, K Zhang, Efficient and robust feature extraction by maximum margin criterion. In Proc. Adv. NIPS, 2004, pp. 157-163.

[24] Lining Zhang, Lipo Wang, and Weisi Lin Semi supervised Biased Maximum Analysis for Interactive Image Retrieval. In IEEE Trans. on Image processing, Vol. 21, No. 4, pp no. 2294-2308, April 2012.

[25] Neetesh Gupta, Dr R.K. Singh, P. K. Dey A New Approach to CBIR using Image Classifier. In IJCA Vol 14, Issue 5 , pp $14-18,2011$.

[26] http://en.wikipedia.org/wiki/Sobel_operator 